\title{
Towards BCl-based Interfaces for Augmented Reality: Feasibility, Design and Evaluation
}

\author{
Hakim Si-Mohammed, Jimmy Petit, Camille Jeunet, Ferran Argelaguet, Fabien Spindler, Andéol Évain, \\ Nicolas Roussel, Géry Casiez, and Anatole Lécuyer
}

\begin{abstract}
Brain-Computer Interfaces (BCls) enable users to interact with computers without any dedicated movement, bringing new hands-free interaction paradigms. In this paper we study the combination of $\mathrm{BCl}$ and Augmented Reality (AR). We first tested the feasibility of using BCl in AR settings based on Optical See-Through Head-Mounted Displays (OST-HMDs). Experimental results showed that a $\mathrm{BCl}$ and an OST-HMD equipment (EEG headset and Hololens in our case) are well compatible and that small movements of the head can be tolerated when using the $\mathrm{BCl}$. Second, we introduced a design space for command display strategies based on $\mathrm{BCl}$ in $\mathrm{AR}$, when exploiting a famous brain pattern called Steady-State Visually Evoked Potential (SSVEP). Our design space relies on five dimensions concerning the visual layout of the $\mathrm{BCI}$ menu ; namely: orientation, frame-of-reference, anchorage, size and explicitness. We implemented various $\mathrm{BCl}$-based display strategies and tested them within the context of mobile robot control in AR. Our findings were finally integrated within an operational prototype based on a real mobile robot that is controlled in $\mathrm{AR}$ using a $\mathrm{BCl}$ and a $\mathrm{HoloLens}$ headset. Taken together our results (4 user studies) and our methodology could pave the way to future interaction schemes in Augmented Reality exploiting 3D User Interfaces based on brain activity and BCls.
\end{abstract}

Index Terms-Brain-computer interface, augmented reality, user interface, design space, SSVEP, optical see-through, robot control.

\section{INTRODUCTION}

B RAIN-Computer Interfaces (BCIs) enable the design of novel interaction schemes based directly on brain activity and mental states of the user [1]. A BCI translates the brain activity into "mental commands" that can be exploited in a wide number of applications ranging from assisting people with disabilities or reeducation therapies to entertainment and video games.

In a previous work [2], we conducted a survey on projects combining BCI and Augmented Reality (AR) and observed that such a combination has been rather scarcely studied so far. The main reasons are probably the still limited performance of BCIbased control, the inherent complexity of these two technologies, as well as the difficulty to design effective User Interfaces (UI) based on BCI.

In this paper we study the use of BCIs in Augmented Reality by following a comprehensive methodology addressing the feasibility, design, and evaluation of BCI-based interfaces for AR. Considering the very high number of dimensions of this problem, we propose to focus on: one BCI category (the BCIs based on the brain pattern called Steady-State Visually-Evoked Potential or SSVEP), one AR category (the optical see-through systems), and one task (mobile robot control). Our methodology could then be extended later on to other contexts.

The SSVEP is a specific brain pattern that occurs when the human visual system is stimulated by a periodic flickering stimulation. The brain responds with an activity at the very same frequency in the visual cortical area [3]. When facing multiple

- H. Si-Mohammed, Jimmy Petit, F. Argelaguet, F. Spindler and A. Lécuyer are with Univ. Rennes, Inria, IRISA, CNRS, Rennes, France.

E-mail: firstname.lastname@inria.fr

- C. Jeunet is with CNBI, EPFL, Geneva, Switzerland and Univ. Rennes, Inria, IRISA, CNRS, Rennes, France.

- G. Casiez is with University of Lille, Villeneuve d'Ascq, France.

- N. Roussel is with Inria Bordeaux, Bordeaux, France.

- Corresponding Authors : Hakim Si-Mohammed and Anatole Lécuyer targets flickering at different frequencies, it becomes possible to determine which target the user is focusing on by analyzing his/her electroencephalography (EEG) signals (see section 3.1.3). By associating each target with a particular command, it becomes possible to create a BCI with multiple mental commands [4]. However, such brain activities are known to be sensitive to both external and internal noise, meaning that the BCI performance can be affected for instance by electromagnetic interference with other equipment (e.g. battery) and/or by the muscular activity of the user (e.g. head movements).

A first objective of this paper is therefore to study the feasibility of combining BCI and AR technologies. Our intention is to measure the influence of both external and internal noise on the performance of an SSVEP-based BCI in AR. We conducted two user studies, assessing the potential drops in BCI performance due to: 1) the wearing of both an EEG headset and an AR headset (study 1); and 2) the movements of the user's head when observing the AR scene (study 2).

A second objective is to propose a generic methodology to design User Interfaces for BCIs based on SSVEP, i.e., based on the selection of flickering targets in AR. We considered, as a starting use-case, the context of robot control, using 3 commanueds, based on SSVEP brain pattern. We present an extended design space of different possible layouts, to display 3 targets for SSVEP-based robot control. This design space comprises five dimensions of 2 modalities: orientation (frontal vs. transversal), frame-of-reference (ego-centered vs. exo-centered), anchorage (user-attached vs. robotattached), size (absolute vs. adaptive) and explicitness (explicit vs. implicit). This design space implies 32 ( $2^{5}$ combinations) different display strategies. We then conducted a user study (Study 3) to select the preferred strategies among 32 which enables to rank them and identify the most intuitive candidates. Following that, we evaluated 4 representative strategies (Study 4), in terms of BCI performance on a concrete case of robot control. 
The third objective of this paper is to illustrate the development of a prototype of BCI-based application in AR. We could integrate all our previous results into a unique setup dedicated to the control of a real mobile robot in AR by means of an SSVEP-based BCI.

We believe that the general method presented in this paper could then guide future research and developments towards effective BCIbased interfaces for augmented reality applications.

The remainder of the paper is structured as follows: Section 2 presents previous works on combining AR and BCIs. Section 3 describes the experimental protocols and the results of two feasibility studies assessing the compatibility of BCI and AR technologies. Section 4 presents our extended design space of commands display strategies for robot control in AR, together with two user studies aiming at identifying the preferred design strategies as well as their influence on BCI performance. Section 5 describes the development of our final prototype for real mobile robot control. The paper ends with a general discussion (Section 6) and a conclusion (Section 7).

\section{Related Work}

The number of works exploring the use of brain-computer interfaces in augmented reality applications remains relatively small [2]. Although preliminary studies suggest it is possible to combine BCI with AR [5], [6], we are not aware of any study systematically investigating the ability to combine AR and BCI.

In this section we summarize the state of the art of using and testing BCIs in AR contexts. We classify the previous works according to the type of AR system they use: (1) Video-See Through, or (2) Optical See-Through.

\subsection{Video See-Through $A R$ and $B C l$}

Video See-Through (VST) AR systems consist in recording real images with camera (tablet, phone, etc.) and redisplay them in real-time augmented with virtual elements.

Using SSVEP in the context of a VST AR has been preliminarily tested by Faller et al. in [5], [6] on 3 subjects. They used a VST system consisting in a HMD and a head-mounted camera, along with a fiducial marker tracking system. The task consisted in navigating a virtual avatar through a predefined path while holding stops at specific platforms using 4 commands: (1) turning $45^{\circ}$ to the right, (2) turning $45^{\circ}$ to the left, (3) walking one unit straight ahead and (4) switching on or off the other stimuli. Two out of the three subjects managed to complete the slalom within the specified time limit. Although this study is promising, the small number of participants as well as the limited performance measurement, makes it important to lead more extensive and formal studies.

In a close context, Gergondet et al. [7] developed an SSVEPbased system to steer a robot in which a subjective view from the robot was displayed on a computer screen augmented with directional and speed controls. As the commands were displayed on a computer screen, the setup did not allow for much mobility. In addition, the first-person view of the robot makes it difficult to determine the precise situation of the robot in its environment. Later, this work was extended to make use of a HMD [8]. In a similar setup, Escolano et al. [9] made use of a the P300 paradigm.

Lenhardt and Ritter [10] developed a P300 ${ }^{1}$-based system to control a robotic arm with a VST-HMD to make it move cubes

1. a specific EEG positive wave appearing approx. $300 \mathrm{~ms}$ after a rare and expected event [11] placed on a table. The cubes were tagged with fiducial markers, and when they were detected by the system, flickering 3D numbers appeared on top of them. By focusing on the number corresponding to the desired cube, the users could select which one to move. After the cube selection, a virtual grid appeared on the table. Each cell of this grid also flickered to represent a potential target area to move the cube.

The Combination of BCIs and AR can also be applied for the medical field. The ViLimbs system designed by Correa-Agudelo et al. [12] for phantom limb pain treatment is a representative example. Using a fiducial marker on the proximal part of the missing arm, this system displays the mirror view of the patient on a large screen, augmented with a virtual limb in place of the missing one. EEG signals were then used to detect attempted movements, allowing the patient to move his arm away from painful positions. This setup can be considered as an enhancement of the mirror therapy for phantom limb pain treatment [13].

It seems that VST-AR is the most used type of AR that has been combined with BCIs. However, one of the leading trends in HMD based AR technologies today, is represented by Optical See-Through AR.

\subsection{Optical See-Through AR and BCl}

Optical See-Through (OST) AR systems, consist in displaying virtual content directly in the users' field of view, onto a semitransparent screen that allows to see the real environment at the same time.

The use of BCIs has been less explored in the context of OSTAR. To the authors' best knowledge, only Takano et al. [14] have explicitly explored this solution. Their study evaluated a home automation system in two steps.

First they conducted a performance evaluation on combining a monocular OST headset with an EEG. They compared the online and offline classification accuracy of P300 selection on a LCD monitor and the monocular HMD. Fifteen subjects were asked to issue five P300 commands through a menu either superimposed in their field of view or displayed on a LCD screen. They reported an online classification accuracy of $88 \%$ on the LCD screen and $82 \%$ on the HMD for TV control. A two-way repeated ANOVA on offline data showed a significant drop of performance between the LCD and the HMD for the same task. They suggested this difference could be due to the different icons' size and distance between the two conditions. Although these results were promising, the classifiers have only been tested under static conditions with no movement and the display conditions were not mentioned as being the same in the two configurations both in terms of focal distance and refresh rate.

Secondly, they tagged home appliances (lamps, televisions) with fiducial markers so that participants could interact by staring at them (using a monocular HMD). When in the user's field of view, the interaction was based on a P300 menu (items structured in a grid layout) representing the different possible actions on the appliance.

The main conclusions from the study of the literature are that most of the developed systems combining AR and BCI rely on Video See-Through (VST), e.g. [7], [10]. Very few researchers have explored the use of BCI with Optical See-Through AR (OST) [14] and none went beyond assessing the mere hardware compatibility between the devices. It is also interesting to note that all the prototypes and the studies relied on simple user interfaces, and 
did not consider the possibility to improve the usability through different layouts for the SSVEP or P300 commands.

In the following sections, we address the question of the different possible sources of disruption as well as the question of the commands display strategies in AR.

\section{Feasibility Study: Testing the compatibil- ITY OF BCI AND AR TECHNOLOGIES}

BCIs are known to be very noise-sensitive. Combining BCI with AR might introduce 2 different sources of disruption. Firstly, at the hardware level, both technologies can require head mounted devices and it is necessary to make sure that they do not interfere (external noise). Secondly, recording brain activity in the context of AR where users are generally free to move may also be difficult as muscle activity can provoke artifacts in the BCI recordings (internal noise).

Therefore, in order to assess the compatibility of a BCI with an OST-HMD AR system, we conducted 2 user studies. The first study, on "external noise", aimed at evaluating the effect of associating the two headsets (BCI and AR headsets) on the performance. The second study, on "internal noise", aimed at evaluating the effect of head movement on the BCI performance. In the scope of this paper we focus on the use of SSVEP in AR.

Before presenting the details of the experiments, it is interesting to point out that, in the literature, SSVEP responses have been reported to be successfully classified with an accuracy around $80 \%$ on screen displayed targets [15], [16] and led-based stimulations [17] in static contexts. Few papers addressed the issue of SSVEP selection in motion [18]. Previous studies reported a deterioration in the SSVEP classification accuracy proportional to the walking speed, but they did not consider the movement of the head which is potentially more disruptive for SSVEP due to the fact that the neck is closer to the occipital lobe (where EEG sensors are typically placed for SSVEP).

The experiments presented in this paper were all conducted in accordance with the relevant guidelines for ethical research according to the Declaration of Helsinki. All the participants were briefed about the nature of the experiment and signed an informed consent form at the beginning of each experiment.

\subsection{User Study 1: Influence of AR Device on BCl perfor- mance}

The objective of this first study was to answer the following questions: (1) is EEG-signal quality impaired due to the wearing of an OST-HMD? (2) Does the electrical activity produced by the display corrupt the signals? (3) Is the SSVEP paradigm effective in such a stereoscopic AR context? These questions are assessed comparing the BCI performance while interacting with an SSVEP interface in different hardware configurations.

In the following paragraphs, the experimental protocol and the results of this study are presented and discussed.

\subsubsection{Apparatus and participants}

Visual stimuli (SSVEP targets) were displayed either on a 47-inch screen or on a Microsoft HoloLens (see Figure 1). Both display devices had the same framerate of $60 \mathrm{~Hz}$. In order to keep a similar configuration between experimental configurations, participants were sat at 2 meters from the screen due to the focal distance of the HoloLens. Furthermore, the screen covered the same area of the participants' central field of view as the HoloLens.

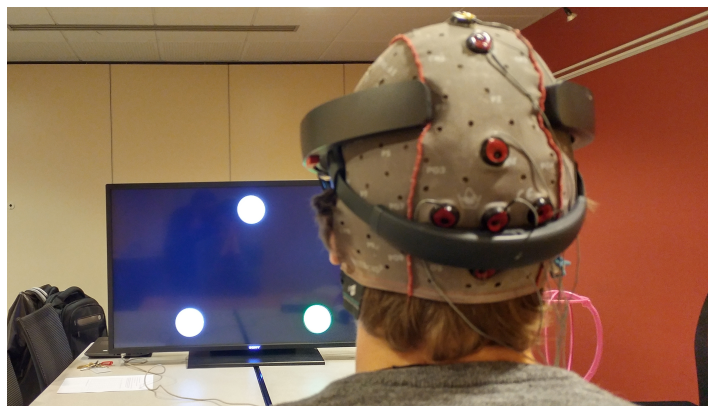

Fig. 1: Experimental setup of Study 1: the user wears an EEG cap as well as the HoloLens. In this particular condition, the SSVEP targets were displayed on the screen.

Electroencephalography (EEG) data were recorded from a g.USBamp amplifier (g.tec, Graz, Austria) using 6 scalp electrodes (CPz, POz, O1, O2, Oz, Iz - 10-20 system) referenced to the ear and grounded to $\mathrm{Cz}$. Such electrode positions enable the coverage of the visual cortex, from where SSVEP are triggered [19].

Thirteen naive participants (aged 25.9 \pm 3.1 year-old, 2 women) took part in the experiment.

\subsubsection{Experimental Protocol}

After they signed the informed consent form and were provided with all the relevant information about the experiment, participants were equipped with the EEG cap.

Following the installation, participants were asked to complete 4 runs of SSVEP training. Each run lasted around 7 minutes (30 trials). Each run consisted in the display of 3 white targets either on the screen or through the HoloLens. The choice of the stimulation frequencies was made according to the literature, reporting which frequencies elicit the highest SSVEP responses [20]. Each of these targets was flickering at a different frequency $(10,12$ and $15 \mathrm{~Hz})$. The targets were $10 \mathrm{~cm}$ wide circles arranged in an equilateral triangle of $46 \mathrm{~cm}$ side.

Participants were instructed to focus on one specific target through the appearance, for $1 \mathrm{~s}$, of an arrow pointing at it. After $7 \mathrm{~s}$, the targets stopped flickering and participants were provided with a feedback: one of the targets was highlighted in green for $2 \mathrm{~s}$. Given the fact that we did not perform any online classification, the feedback was sham. The correct target was highlighted $80 \%$ of the time while one of the other two was highlighted the rest of the time in order to keep the participants motivated. Then, participants had a $2 \mathrm{~s}$ break before the next trial started. The structure of the trials is illustrated in Figure 2.

In total, participants had to focus on each target 10 times per run, in a random order. The session was divided into 4 runs, with one run per condition: (C1) targets displayed on screen, no HoloLens, (C2) targets displayed on screen, HoloLens switchedoff, (C3) targets displayed on screen, HoloLens switched-on, (C4) targets displayed through the HoloLens. The order of the runs was randomized across participants to avoid any potential order effect. In total, the experiment consisted in 120 trials for a duration of 22 minutes.

The classification accuracy for each condition provides insights about the potential impact of the OST-HMD on the EEG data quality and on the viability of using SSVEP in a stereocopic AR context. Our hypothesis was that the classification accuracy would 


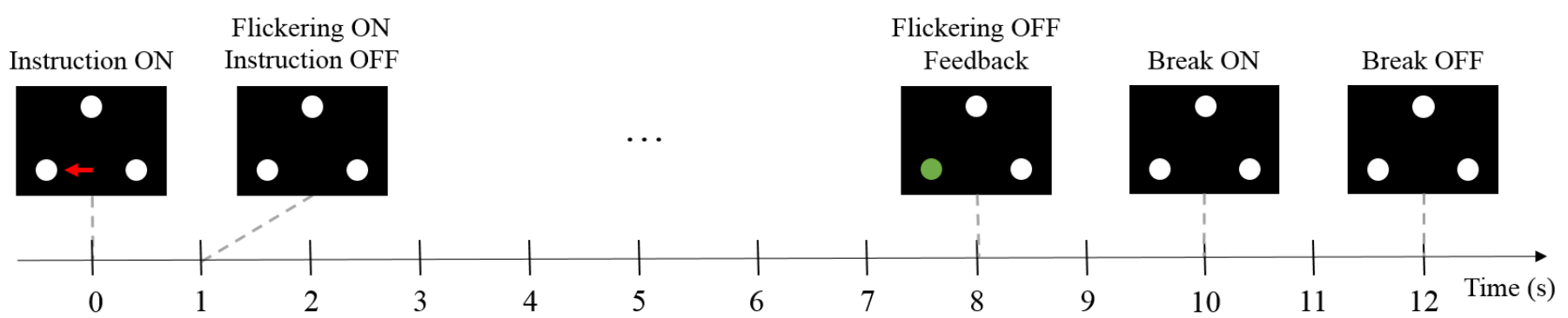

Fig. 2: Trial timing. $(\mathrm{t}=0 \mathrm{~s})$ The user is instructed to focus on a particular target through an arrow. ( $\mathrm{t}=1 \mathrm{~s})$ The arrow disappears and all targets flicker for $7 \mathrm{~s}$. ( $\mathrm{t}=8 \mathrm{~s})$ The flickering stops and a sham feedback is provided (the designated target is highlighted in green in $80 \%$ of the trials). ( $\mathrm{t}=10 \mathrm{~s})$ A two-second break starts before the next trial begins.

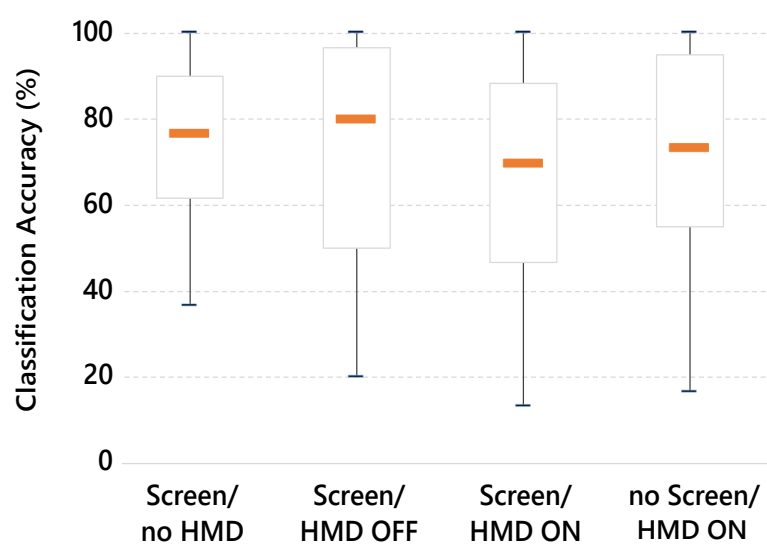

Fig. 3: Results of Study 1: Boxplots representing the classification accuracy in \% $(\mathrm{N}=13)$ as a function of the visual condition.

not be significantly different depending on the condition, i.e., that participants would perform the same in the four conditions.

\subsubsection{Offline EEG Data Analysis}

Data were sampled at $512 \mathrm{~Hz}$ and processed using the OpenViBE software [21]. As stated before, the targets were flickering at 10, 12 and $15 \mathrm{~Hz}$. There were 10 trials per class (each flickering frequency corresponds to a class) per run, i.e., 30 trials per run in total. To determine the performance obtained at each run, we performed an offline 10-fold cross-validation procedure. Thus, for each class of each run, 9 trials were used to train the multiclass Common Spatial Pattern (CSP [22]) and the Linear Discriminant Analysis (LDA [23]) algorithms while the last trial was used to test these algorithms. This operation was computed 10 times for each class of each run. The multiclass CSP consisted in filtering the data in $[9.75 ; 10.25] \mathrm{Hz}$ for the $10 \mathrm{~Hz}$ class, $[11.75 ; 12.25] \mathrm{Hz}$ for the $12 \mathrm{~Hz}$ class and $[14.75 ; 15.25] \mathrm{Hz}$ for the $15 \mathrm{~Hz}$ class and then in finding 6 spatial filters whose resulting EEG power was maximally different for one class vs. the others. The spatially filtered EEG signal power (computed on a 500ms time window, with $100 \mathrm{~ms}$ overlap) was used to train a multiclass LDA. This LDA was then used to discriminate when the user was focusing on the targets flickering at 10,12 or $15 \mathrm{~Hz}$.

\subsubsection{Results and Discussion}

One of the samples did not follow a normal distribution. Therefore, we performed a non-parametric test for paired-samples, i.e., a
Friedman test, to test this hypothesis. Given the relatively high number of samples (4 runs and 13 participants), this test could be approximated by the $\chi^{2}$ test with 3 degrees of freedom (4 runs -1 ). In line with our hypothesis, results showed no significant difference between the runs $\left[\chi^{2}(3)=5.025 ; p=0.170\right]$. Results are summarized in Figure 3.

The classification accuracy tended to confirm our hypothesis as all of the conditions' classification accuracy were between $75 \%$ and $80 \%$ which is consistent with the literature [15], [16]. It also confirms the previous results of Takano et al. [14] on the possibility to combine AR and BCI headsets.

Concerning user experience, participants reported that the HoloLens felt heavy after a while. Also, they reported some reflections when it was switched-on. These reflections appeared to be uncomfortable for some of them when focusing on the targets on the screen.

\subsection{User Study 2: influence of Users' Movements on $\mathrm{BCl}$ Performance in AR}

One of the main advantages when using an OST-HMD, is the possibility for the user to move while interacting with the system. Particularly, the user generally has to move his/her head in order to interact with the AR environment.

Thus, the objective of this second study was to evaluate the impact of head movements (source of internal noise) on the BCI selection performance in order to answer the questions: Is it possible to perform a SSVEP selection on moving targets in AR? Is head movement influencing the BCI performance? For this matter, we use the notion of movement intensity designating a combination of amplitude and speed. A low-intensity movement is performed at low speed and low amplitude while a high-intensity movement is fast and wide. In order to assess the possibility of using SSVEPbased BCIs on moving targets, we compared user performance on selecting targets over different movement intensities.

The following paragraphs detail the implemented experimental protocol as well as the results of the experiment.

\subsubsection{Apparatus and Participants}

Visual stimuli were displayed again on a Microsoft Hololens. The EEG apparatus was the same as in the previously described experiment. In order to ensure consistency over the results of the participants, they were asked to stand up on the same spot and only to move their head to follow the moving targets. In this experiment, we wanted to evaluate a worst real case scenario where the users would not be able to anticipate the movements of the targets, thus 
participants were not informed of the direction the targets would follow.

Fifteen participants (aged $22.8 \pm 2.6$ year-old, 1 woman) took part to the experiment.

\subsubsection{Experiment Protocol}

After the installation, participants were asked to perform 4 runs of SSVEP training. Each run lasted around 7 minutes and consisted of 36 trials. In this case, a trial consisted in the display of 3 moving white targets through the HoloLens. Each of these targets was flickering at a different frequency $(10,12$ and $15 \mathrm{~Hz})$. They were represented as $10 \mathrm{~cm}$ wide circles arranged in an equilateral triangle of $46 \mathrm{~cm}$ side in order to keep the same configuration as the previous study. Similarly, the structure of a trial and the feedback strategy were also identical to the one described in Figure 2.

During each run, participants had to focus on each target 12 times under a different configuration of movements, submitted in a random order. There were 4 conditions of head movements: (C1) A test condition with no movement (static targets), (C2) a low-intensity movement configuration with a maximum amplitude from left to right of $40^{\circ}$, (C3) a medium-intensity movement configuration with a maximum amplitude of $100^{\circ}$ and (C4) a highintensity movement with a maximum amplitude of $160^{\circ}$. In this case, the movement amplitude represents the rotation angle of the head.

The chosen movement form (path of the targets) was a lemniscate of Bernoulli (see Figure 4). In addition to being symmetric, this path contains different horizontal and vertical orientations of movement. The lemniscate of Bernoulli is smooth and prevents from sudden directions changes, reducing potential artifacts arising from discontinuity. The direction to perform the path was counterbalanced across each class. As the user had to focus on each class 12 times, he/she had to perform the path 3 times from each of the 4 possible initial movements i.e. beginning from (1) top right, (2) top left, (3) down right and (4) down left. This counterbalancing was done to avoid biases that might have appeared due to the repetitive execution of the same head movement which could have biased the classification.

Finally, using the parametric representation (Equation 1), it was possible to implement different movement amplitudes by changing the half focal distance of the lemniscate $d_{i}$ (see Figure 4) while ensuring to perform the whole movement in 7 seconds, implementing the notion of movement intensity. In other words, a small lemniscate was performed in 7s (at low speed) and a larger one also in 7s (at a higher speed).

$$
\left\{\begin{array}{l}
x(t)=d \sqrt{2} \frac{\sin \psi(t)}{1+\cos ^{2} \psi(t)} \\
y(t)=d \sqrt{2} \frac{\sin \psi(t) \cos \psi(t)}{1+\cos ^{2} \psi(t)}
\end{array} \text { with } \psi(t)=2 \pi \frac{t}{7}-\pi(1)\right.
$$

\subsubsection{Results and Discussion}

According to the results of the literature [18], [24], our hypothesis was that head movement would deteriorate the classification accuracy of SSVEP responses. Particularly as this paradigm is exploited from recordings on the visual cortex at the back of the head, and as head movements solicit the neck, the strongest muscle artifacts should appear in that area, close to the EEG electrodes. Accordingly, the results of the experiment show a clear degradation of the BCI performance together with the increase in movements

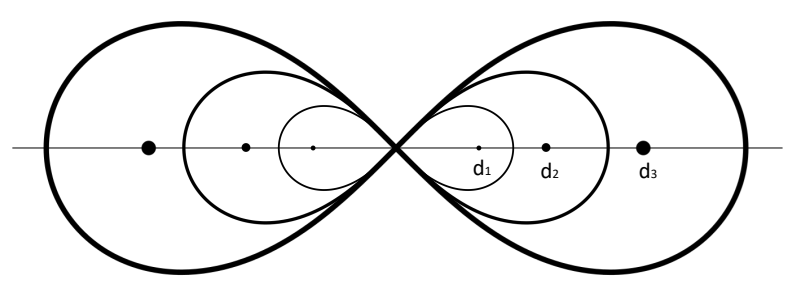

Fig. 4: Different Movement intensities and targets motion used in user study 2: Lemniscates of Bernoulli with half focal distances $d_{1}\left(40^{\circ}\right)<d_{2}\left(100^{\circ}\right)<d_{3}\left(160^{\circ}\right)$ The path of the targets lays in a sphere centered on the user.

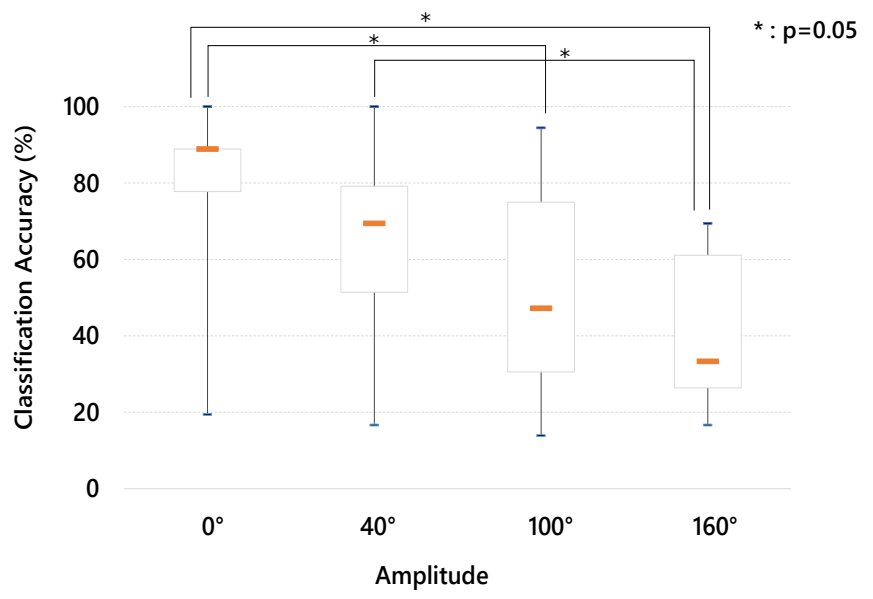

Fig. 5: Results of Study 2: Boxplots representing the classification accuracy in $\%(\mathrm{~N}=15)$ as a function of the movement intensity.

intensity (see Figure 5). Thus, average performance drops from $78 \%$, when participants do not move, to $41 \%$ (which is around chance level [25]) for the maximal movement amplitude.

Moreover, performing a statistical test on the data showed that this difference was statistically significant at $95 \%$ of confidence (Friedman test was performed as in section 3.1.4). However, after performing Post-Hoc test through multi-comparisons between conditions after Friedman test, it appeared that the difference between the "No Movement" and the "Low Intensity Movement" conditions was not significant (see Figure 5).

In addition to the statistical analysis, a study of the forms filled by the participants right after the experiment, revealed that a large majority of participants had difficulties following the targets in the "High Intensity Movement" condition considering it an extreme condition. Thus, the deterioration of performance could also be partially due to the inability to accurately track the targets.

In a nutshell, the results of this experiment suggest that it is possible to use SSVEP as a selection technique in the context of AR with small head movements (around $40^{\circ}$ of amplitude). They also show a significant deterioration of performance with higher intensity movement $\left(100^{\circ}\right)$ but still above chance level [25], whereas extremely high intensity movement prohibits the use of SSVEP. 


\section{A DESIGN SPACE FOR BCI-BASED USER INTER- FACES IN AR: SSVEP TARGETS DISPLAY STRAT- EGY FOR ROBOT CONTROL}

When designing interactive systems in AR based on BCIs, in addition to the classification performance, the User Interface plays an important role for the usability of the system. In the case of SSVEP, the UI lies in the layout of a selection menu and the strategy to display several targets (typically 3 ) to the user. This layout however, is strongly related to the task and the application that it is designed for. As it is important that the user seamlessly understands the meaning of the commands in the context of the application, the coherent display and integration of command buttons in the interface highly depends on the task. In the case of this paper, we chose the illustrative example of a robot teleoperation system.

Therefore, in this section we conceive a design space of all possible layouts for the integration of 3 SSVEP targets in an AR environment, for the control of a mobile robot.

We believe that our approach, our methodology and our design space could be extended to other contexts, e.g. more targets, other BCI paradigms, other tasks etc.

\subsection{Design Space of SSVEP Target Display Strategies}

Our objective is here to propose a design space that would characterize all the possibilities to display 3 SSVEP targets in the 3D space. Considering our application case of mobile robot control, three commands are enough for steering the robot with 3 options: "Forward", "Turn Left" and "Turn Right". In order to propose the most coherent association of each command with the AR target, we propose a 5-dimension design space that describes all possible layouts of 3 command targets. These 5 dimensions, namely Orientation, Frame-of-reference, Anchorage, Size and Explicitness, are presented beneath:

- Orientation: This dimension corresponds to the orientation of the plane containing the 3 targets. This plane can be "Transversal" i.e. parallel to the horizon or "Frontal" i.e. facing the user (see Figure 6a). When arranged transversally, the targets are in the same plane as the robot is moving. We hence expect that it would be easier to associate every command with its corresponding effect. When arranged frontally, the user has to make a mental rotation along the horizontal axis.

- Frame-of-reference: This dimension corresponds to the frame of reference of the 3 targets. It can be set to the robot, or to the user. It can be associated to the concepts of "ego-centered" and "exo-centered" frames of reference. In the first case, the targets correspond to absolute direction, set relatively to the user. For associating a target with a direction command, the user has to perform a mental rotation to map the targets with the robot direction of movement. When "exo-centered" i.e. attached to the robot, the plane containing the targets would rotate with the rotation of the robot and thus, keep the targets' arrangement constant relatively to the robot. In this second setup, the target at the right side of the robot will always correspond to a right turn (see Figure $6 b$ ).

- Anchorage: This dimension corresponds to the position of the targets' frame of reference in 3D. The targets can be either "anchored" to the robot or to the user. In the first

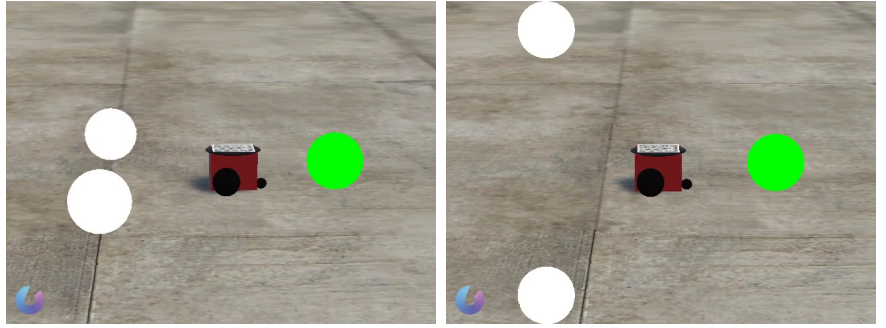

(a) Orientation: Transversal (left), Frontal (right)
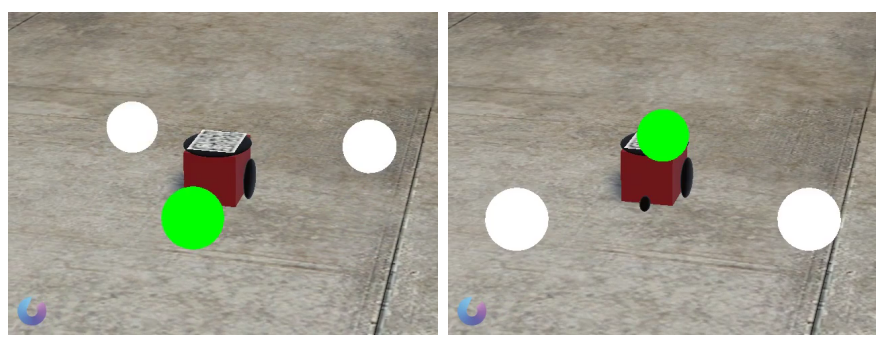

(b) Frame of Reference: Exo-Centered (left), Ego-Centered (right)
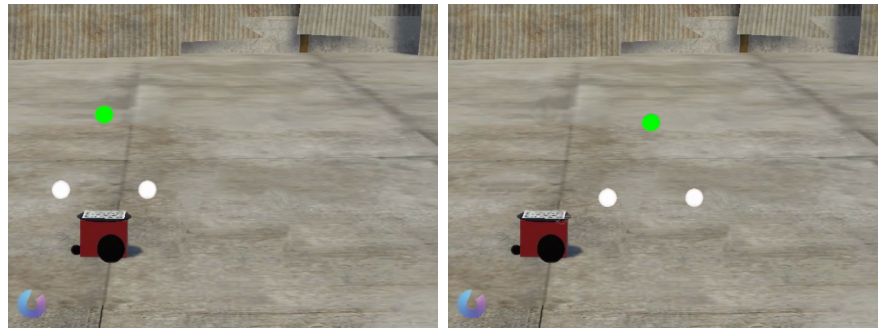

(c) Anchorage: Robot (left), User (Right)
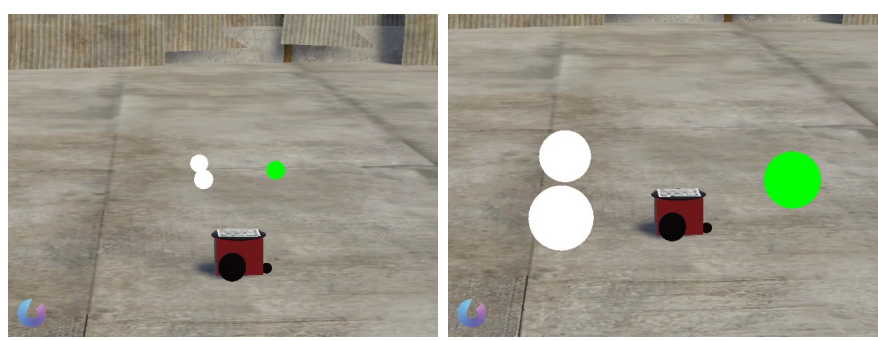

(d) Size: Absolute (left), Adaptive (Right)

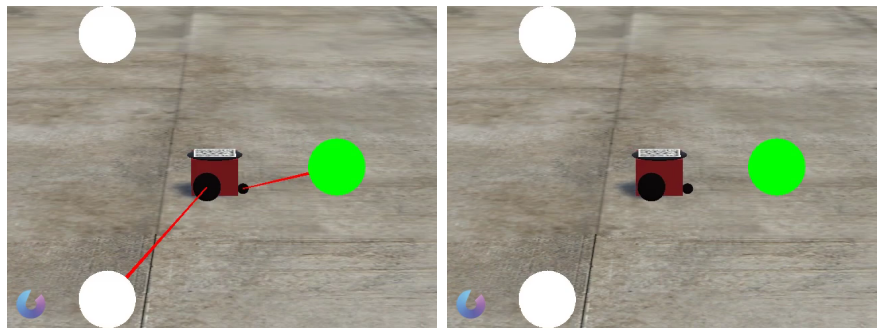

(e) Explicitness: Explicit (Left), Implicit (Right)

Fig. 6: Illustration of the 5 dimensions of our design space of SSVEP targets layout and display strategies. Each couple of figures represent the 2 modalities of 1 dimension: (6a) the orientation of the plane containing the targets, (6b) the frame-of-reference of the targets coordinates, (6c) the anchorage of the targets and their position, (6d) the size of the targets which can be absolute or adaptive and (6e) the explicitness of the association target/command. 
case the three targets are set on the top of the robot and thus move with the robot. In the second case they are attached to the field of view of the user - at a distance of 2 meters to meet the focal distance configuration of the HMD. In such case, they would move accordingly to users' head movements (see Figure 6c). The main difference between the two modalities lies in the fact that when anchored to the user, the latter can issue commands to the robot even when it is no longer in the user's field of view.

- Size: This dimension relates to the size of the targets, which can also be an influential parameter for the system ergonomics. The targets' size can be either "Absolute" or "Adaptive". As the targets are 3D objects, we used this terminology to define their real size. When the size in set to absolute, the radius of the target is fixated in the 3D space. In other words, the farther the robot is from the user, the smaller the targets appear. With the perspective, the size of the targets depends on the distance between the user and the robot. In the adaptive condition, the real size of the targets in the 3D space is not fixated. It would adapt to always be displayed at the same size relatively to the user. In other words, even if the robot is far from the user, the size of targets increases to compensate, and thus the targets always appear at the same size to the user (see Figure 6d). The advantage of the absolute setup lies in the fact that it is easier to associate the targets with the robot they control. However, bigger targets can be more comfortable to focus on compared to smaller ones. Lastly, it has been reported [26] that larger SSVEP targets elicit stronger SSVEP responses, we thus expect better classification results in the configurations where the size is set to adaptive.

- Explicitness: Rather than a display configuration, this dimension corresponds to the explicitness of the association between the targets and the commands. One way to improve the semantics of the application is to explicitly link a target with the direction it leads towards. When the explicitness is set to "Explicit", we chose to link the target with the corresponding wheel of the robot (front wheel, right wheel and left wheel) with a visual red string (see Figure 6e). When set to "Implicit", no red string was displayed. Even though, this additional content may overcharge the user's field of view, we hypothesize that it will help to seamlessly associate the target with the command.

The combination of these 5 dimensions of 2 modalities each (see Figure 6) implies a tree of $32\left(2^{5}\right)$ possible display strategies for our design space. In the following section, we propose qualitative and quantitative evaluations of these display strategies through 2 user studies. In the first one (section 4.2) we study how the users subjectively perceive and prefer the different strategies. In the second user study (section 4.3) we quantitatively evaluate the best ranked ones (per group) in terms of their BCI performance.

\subsection{User Study 3: Subjective Preference of the Targets' Display Strategies}

We conducted a user study in order to evaluate the different display strategies according to their subjective intuitiveness and coherence relatively to the task. Our goal was to determine the command layouts that the user would perceive as the easiest to understand considering the scenario of robot control.

\subsubsection{Experimental Protocol}

We recorded 32 video shots of 24 seconds each, corresponding to all the possible display strategies (reader may refer to accompanying video). All the 32 videos displayed the same virtual mobile robot executing the same path with different display strategies. This path was designed so to have the same number (4) of command occurrences (turning right, turning left and moving forward) in addition to display the robot from the 4 possible angles (left to right, right to left, back to front and front to back) for every strategy. Thus, no particular configuration was over-represented compared to the others, which would have potentially biased the results. Every time a command was issued to the robot, the corresponding target turned to green right before and during the execution of the command, in order to highlight the association between the command and the target.

An online and anonymized questionnaire filled by 42 participants, enabled to compare the different display strategies by asking the participants to evaluate "how coherent/intuitive was the association between the targets and the commands" for each video on a 7-point Likert scale (Reject, Poor, Acceptable, Satisfactory, Good, Very Good and Excellent). The 32 videos were displayed in a fully randomized order for each participant, in order to minimize ordering effects.

The final ranking of the strategies was performed following a majority judgment procedure [27]. This method consists in 3 steps. First, the majority-grade of an item (display strategy) is defined: it corresponds to the median grade voters attributed to this item. Second, the majority-grade should be completed by a + or - sign depending on whether more voters attributed a higher or lower grade to this item, respectively. Finally, the majority-ranking enable to arrange the items according to their majority-grades. In order to perform this majority-ranking, the majority-gauge of each item should be computed. This gauge is composed of three values ( $\mathrm{p}$, $\alpha, \mathrm{q}$ ), where $\alpha$ is the majority-grade and $\mathrm{p} / \mathrm{q}$ are the percentage of grades above/below the majority-grade, respectively.

The videos were named from 0 to 31 according to the binary value of each dimension: $N=O * 2^{4}+F * 2^{3}+A * 2^{2}+$ $S * 2^{1}+E * 2^{0}$ with O,F,A,S,E (standing for Orientation, Frame-ofReference, Anchor, Size and Explicitness dimension respectively). This notation enabled us to interpret the clustering obtained after the evaluation.

\subsubsection{Results and Discussion}

Table 1 represents the results of the majority judgment for each of the 32 videos and display strategies. As a result of this method of ranking, we could cluster the videos in 4 groups according to the values of 2 dimensions ("Orientation" and "Frame-of-reference") (see Figure 1). The group of videos with an exo-centered frameof-reference and with a transversal orientation all have a "good" majority grade evaluation (videos from 25 to 32). The group with frontal orientation and an exo-centered frame-of-reference has a majority judgment of "satisfactory" while the two other groups have a "poor" or "acceptable" dominant majority grade evaluation.

Taken together, our results suggest strong trends regarding the subjective preference of the users. In a nutshell, we found that participants preferred a transversal orientation, meaning that the targets should remain in the same plane as the robot's motion i.e. the horizontal plane here. More importantly, the user strongly preferred when the frame-of-reference for the targets coordinates was set to the robot (exo-centered). We hypothesize that these two 
configurations (transversal orientation and exo-centered frame-ofreference) minimize the mental rotation necessary to map the command with the direction. However, the highest subjective preference of the users does not guarantee the best objective performance of the SSVEP detection. Therefore, in the following section, we describe another user study meant to study the influence of the UI and display strategy on the BCI performance.

\subsection{User Study 4: Influence of the Targets' Display Strategy on BCl Performance}

To quantitatively evaluate the influence of the targets' display strategy on the classification performance, we conducted a user study in which we kept the best ranked and most representative display strategy (DS) from each of the 4 groups of strategies previously obtained (in section 4.2.2). We selected the best strategy from each group, provided that at least 2 dimensions differ between each 2 strategies. This method resulted in the selection of the strategies DS0, DS15, DS22 and DS29 corresponding to:

- DS0: ("Frontally-oriented", "Ego-centered", "Useranchored", "Adaptive-sized" and "Implicit") display strategy (see Figure 7a)

- DS15: ("Frontally-oriented", "Exo-centered", "Robotanchored", "Absolute-sized" and "Explicit") display strategy (see Figure 7b)

- DS22: ('Transversally-oriented", "Ego-centered", "Robotanchored", "Absolute-sized" and "Implicit") display strategy (see Figure 7c)

- DS29: ('Transversally-oriented", "Exo-centered", "Robotanchored", "Adaptive-sized" and "Explicit") display strategy (see Figure 7d)

DS0 is considered here as the control condition, since this display strategy is similar to a standard SSVEP training phase and known to maximize the SSVEP response [26].

\subsubsection{Apparatus and Participants}

We implemented an augmented reality playground with a highlighted path that a virtual robot had to move through as illustrated in figure 8 . This path was designed so that all of the three command directions are used the same amount of times to complete it.

The virtual playground and all its components (path, robot and command targets) were displayed on a Microsoft HoloLens. The EEG headset was the same as described in Section 3. Twentyfour participants (aged 28.1 $\pm 8.1,4$ women) took part to this experiment.

\subsubsection{Experimental Protocol}

After they signed the informed consent and were given all relevant information, the participants were equipped with both the EEG and the AR headsets. The experiment was composed of two parts: (1) a training acquisition phase that lasted $7 \mathrm{~min}$ and that was used to gather the data to train the classifiers, and (2) an evaluation phase that was used to compare the 4 display strategies in terms of BCI performance.

The training phase consisted of a unique run of 30 trials (SSVEP selections). The targets were $10 \mathrm{~cm}$ wide circles arranged in an equilateral triangle of $46 \mathrm{~cm}$ side placed at a distance of $2 \mathrm{~m}$ from the user. They were flickering at 10, 12 and $15 \mathrm{~Hz}$. The trial structure was the same as described in Figure 2.

Following the training phase, the evaluation phase consisted in 4 runs of 18 trials ( 4 min). Each run was using one of the selected
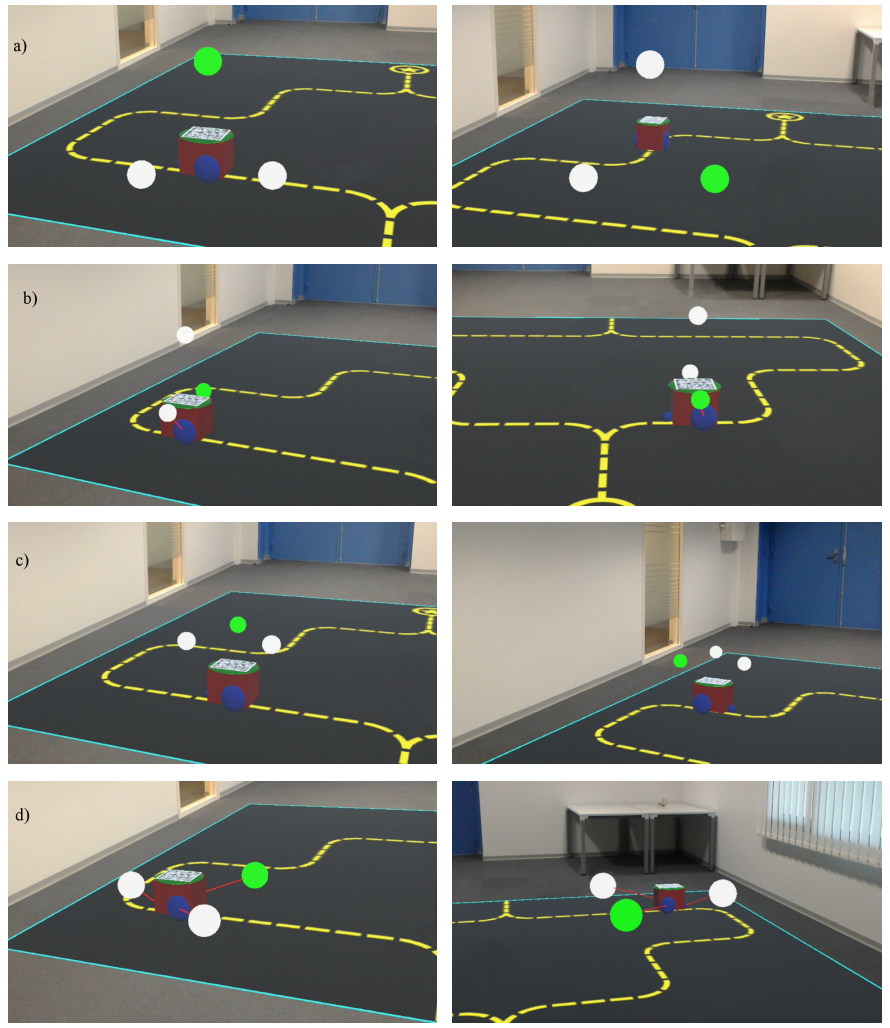

Fig. 7: Illustration of the 4 display strategies used in user study 4. DS0 (a), DS15 (b), DS22 (c), DS29 (d).

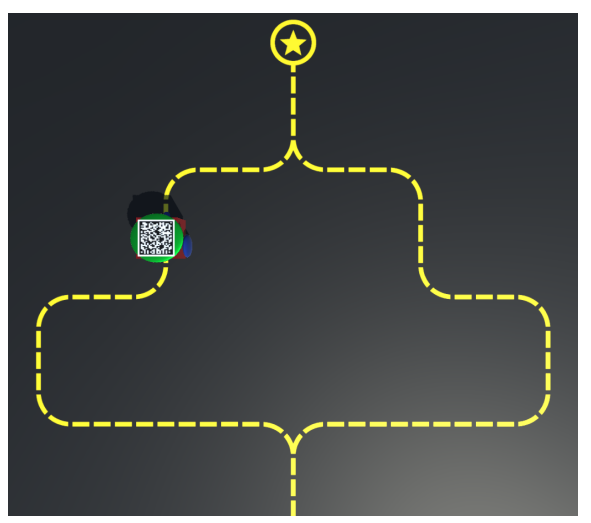

Fig. 8: Virtual playground and highlighted path to follow.

display strategies, and consisted in a round-trip of 9 trials. Each trial consisted in focusing on the designated command target for 7 seconds, followed by 4 seconds of feedback (the recognized target), during which, the robot performed the given action. In order to maximize the consistency between the participants, they only made selections while the robot was immobile. Moreover, the robot always performed the correct move and the feedback was sham. The participants were informed that the robot would always follow the right path, but they were not told about the Sham aspect of the feedback. Hence, to keep a high level of engagement, their objective was to maximize the number of correct detections. In order to avoid any order effect that would bias the results, the order of the runs was counterbalanced across participants ( 24 possible arrangements for 24 participants). 
TABLE 1: Results of user study $3(\mathrm{~N}=42)$. The majority grade corresponds to the median, which means that at least $50 \%$ of the participants evaluated the strategies at least as the majority grade. Four groups, corresponding the 4 possible combinations of the Orientation and Frame-of-reference dimensions, emerge according to the dominant majority grade: poor, satisfactory, acceptable and good for [1-8], [9,16], [17-24] and [24-32] resp. (O: Orientation, FoR: Frame-of-reference, A: Anchorage, S: Size, Exp: Explicitness, T: Transversal, F: Frontal, Ex: Exo-centered, Eg: Ego-Centered, R: Robot, U: User, E: Explicit, I: Implicit.). The color coding of columns $\mathrm{O}$ and $\mathrm{FoR}$ refer to the 4 groups defined by the combinations of the 2 variables.

\begin{tabular}{|c|c|c|c|c|c|c|c|c|c|}
\hline \multirow[t]{2}{*}{ Majority ranking } & \multirow[t]{2}{*}{ Display Strategy \# } & \multicolumn{5}{|c|}{ Dimensions } & \multirow{2}{*}{$\begin{array}{l}\mathrm{p} \% \text { below the majority-grade } \\
\text { a }\end{array}$} & \multirow{2}{*}{$\alpha \pm$ majority-grade } & \multirow[t]{2}{*}{ q \% above majority-grade } \\
\hline & & $\mathrm{O}$ & FoR & A & $\mathrm{S}$ & Exp & & & \\
\hline 1 & 29 & $\mathrm{~T}$ & Ex & $\mathrm{R}$ & Ad & $\mathrm{E}$ & $48 \%$ & Good+ & $17 \%$ \\
\hline 2 & 30 & $\mathrm{~T}$ & Ex & $\mathrm{R}$ & Abs & I & $45 \%$ & Good+ & $31 \%$ \\
\hline 3 & 31 & $\mathrm{~T}$ & Ex & $\mathrm{R}$ & Abs & $\mathrm{E}$ & $43 \%$ & Good+ & $29 \%$ \\
\hline 4 & 28 & $\mathrm{~T}$ & Ex & $\mathrm{R}$ & Ad & I & $40 \%$ & Good+ & $31 \%$ \\
\hline 5 & 26 & $\mathrm{~T}$ & Ex & $\mathrm{U}$ & Abs & I & $31 \%$ & Good+ & $29 \%$ \\
\hline 6 & 24 & $\mathrm{~T}$ & Ex & $\mathrm{U}$ & Ad & I & $33 \%$ & Good- & $33 \%$ \\
\hline 7 & 27 & $\mathrm{~T}$ & Ex & $\mathrm{U}$ & Abs & $\mathrm{E}$ & $31 \%$ & Good- & $43 \%$ \\
\hline 8 & 25 & $\mathrm{~T}$ & Ex & $\mathrm{U}$ & Ad & $\mathrm{E}$ & $21 \%$ & Good- & $43 \%$ \\
\hline 9 & 15 & $\mathrm{~F}$ & Ex & $\mathrm{R}$ & Abs & $\mathrm{E}$ & $33 \%$ & Good- & $45 \%$ \\
\hline 10 & 8 & $\mathrm{~F}$ & Ex & $\mathrm{U}$ & Ad & I & $26 \%$ & Good- & $50 \%$ \\
\hline 11 & 9 & $\mathrm{~F}$ & Ex & $\mathrm{U}$ & Ad & $\mathrm{E}$ & $48 \%$ & Satisfactory+ & $33 \%$ \\
\hline 12 & 10 & $\mathrm{~F}$ & Ex & $\mathrm{U}$ & Abs & I & $45 \%$ & Satisfactory+ & $33 \%$ \\
\hline 13 & 11 & $\mathrm{~F}$ & Ex & $\mathrm{U}$ & Abs & $\mathrm{E}$ & $43 \%$ & Satisfactory+ & $36 \%$ \\
\hline 14 & 14 & $\mathrm{~F}$ & Ex & $\mathrm{R}$ & Abs & I & $38 \%$ & Satisfactory- & $43 \%$ \\
\hline 15 & 12 & $\mathrm{~F}$ & Ex & $\mathrm{R}$ & Ad & I & $36 \%$ & Satisfactory- & $50 \%$ \\
\hline 16 & 0 & F & Eg & $\mathrm{U}$ & Ad & I & $31 \%$ & Satisfactory- & $50 \%$ \\
\hline 17 & 13 & $\mathrm{~F}$ & Ex & $\mathrm{R}$ & Ad & $\mathrm{E}$ & $48 \%$ & Acceptable+ & $31 \%$ \\
\hline 18 & 16 & $\mathrm{~T}$ & $\mathrm{Eg}$ & $\mathrm{U}$ & Ad & I & $38 \%$ & Acceptable- & $43 \%$ \\
\hline 19 & 22 & $\mathrm{~T}$ & $\mathrm{Eg}$ & $\mathrm{R}$ & Abs & I & $33 \%$ & Acceptable- & $43 \%$ \\
\hline 20 & 2 & $\mathrm{~F}$ & $\mathrm{Eg}$ & $\mathrm{U}$ & Abs & I & $26 \%$ & Acceptable- & $45 \%$ \\
\hline 21 & 1 & $\mathrm{~F}$ & $\mathrm{Eg}$ & $\mathrm{U}$ & Ad & $\mathrm{E}$ & $38 \%$ & Acceptable- & $50 \%$ \\
\hline 22 & 7 & $\mathrm{~F}$ & $\mathrm{Eg}$ & $\mathrm{R}$ & Abs & $\mathrm{E}$ & $24 \%$ & Acceptable- & $50 \%$ \\
\hline 23 & 4 & $\mathrm{~F}$ & $\mathrm{Eg}$ & $\mathrm{R}$ & Ad & I & $45 \%$ & Poor+ & $24 \%$ \\
\hline 23 & 6 & $\mathrm{~F}$ & $\mathrm{Eg}$ & $\mathrm{R}$ & Abs & I & $45 \%$ & Poor+ & $24 \%$ \\
\hline 25 & 20 & $\mathrm{~T}$ & $\mathrm{Eg}$ & $\mathrm{R}$ & Ad & I & $45 \%$ & Poor+ & $26 \%$ \\
\hline 25 & 5 & $\mathrm{~F}$ & $\mathrm{Eg}$ & $\mathrm{R}$ & Ad & $\mathrm{E}$ & $45 \%$ & Poor+ & $26 \%$ \\
\hline 27 & 17 & $\mathrm{~T}$ & $\mathrm{Eg}$ & $\mathrm{U}$ & Ad & $\mathrm{E}$ & $45 \%$ & Poor+ & $36 \%$ \\
\hline 28 & 18 & $\mathrm{~T}$ & $\mathrm{Eg}$ & $\mathrm{U}$ & Abs & I & $45 \%$ & Poor+ & $38 \%$ \\
\hline 29 & 3 & $\mathrm{~F}$ & $\mathrm{Eg}$ & $\mathrm{U}$ & Abs & $\mathrm{E}$ & $40 \%$ & Poor+ & $38 \%$ \\
\hline 30 & 21 & $\mathrm{~T}$ & $\mathrm{Eg}$ & $\mathrm{R}$ & Ad & $\mathrm{E}$ & $33 \%$ & Poor- & $36 \%$ \\
\hline 31 & 23 & $\mathrm{~T}$ & $\mathrm{Eg}$ & $\mathrm{R}$ & Abs & $\mathrm{E}$ & $36 \%$ & Poor- & $38 \%$ \\
\hline 32 & 19 & $\mathrm{~T}$ & $\mathrm{Eg}$ & $\mathrm{U}$ & Abs & $\mathrm{E}$ & $38 \%$ & Poor- & $45 \%$ \\
\hline
\end{tabular}

\subsubsection{Results and Discussion}

Following the results of the literature [26], our hypothesis was that the participants would perform the best in DS0 condition as it optimizes the size of the targets and the distance between them. In order to compare the SSVEP recognition performance between all the conditions (DS0, DS15, DS22 and DS29), we used the data gathered during the training phase to train the CSP filters and the LDA classifiers for each participant. Every 7s trial was subdivided into $500 \mathrm{~ms}$ epochs, with an overlap of $400 \mathrm{~ms}$. Each epoch was classified and majority voting across all the epochs determined the recognized class.

Our analysis considered the percentage of correct responses for the entire run (see Figure 9). We removed the data from 3 participants as their results were below the chance level which represents less than $20 \%$ of the population, this observation was consistent with the literature about BCI illiteracy [28]. As the percentage of correct responses did not follow a normal distribution we performed a Friedman test approximated by the $\chi^{2}$ test with 3 degrees of freedom (4 runs -1$)$. The Friedman test showed a significant effect of the display strategy $\left[\chi^{2}(3)=12.23 ; p<0.01\right]$.

After pairwise comparisons using the Wilcoxon signed rank test, on the effect of the condition on the results, we observed a significant difference between the conditions DS0 and DS15 [ $p<$
$0.05]$ and between DS0 and DS22 [ $p<0.05]$ but not significant between DS0 and DS29. In addition, the mean results of the participants were above chance level (45\% [25]) for all of the evaluated display strategies.

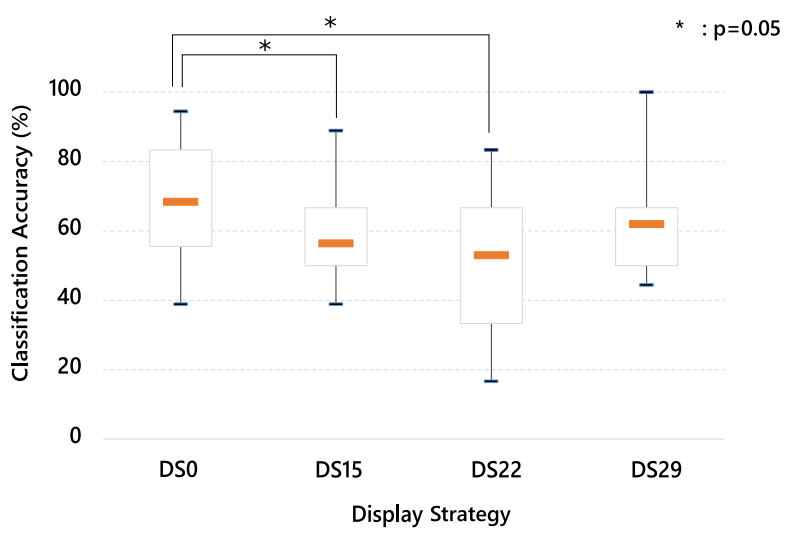

Fig. 9: Results of User Study 4: Boxplots representing the classification accuracy, in \%, (N=21) as a function of the display strategy. 


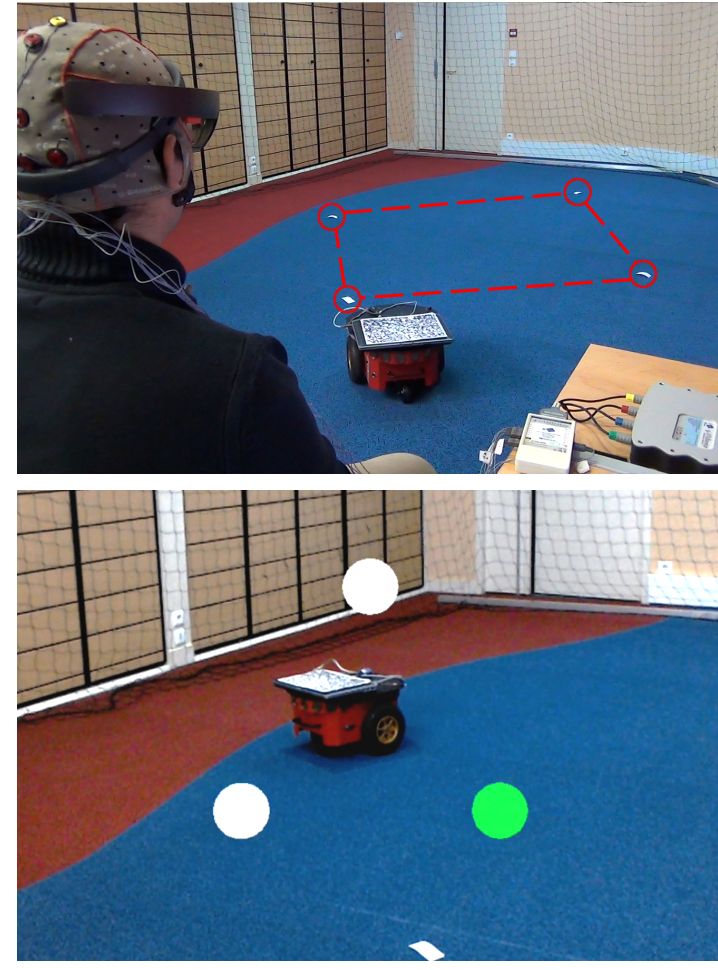

Fig. 10: Illustration of our final prototype in use. (Top) general overview of the setup with the user equipped with EEG, sitting and facing the real mobile robot. (Bottom) First person view, as seen from the HoloLens using display strategy DS0. The dashed line represents the path that the robot moved through during testing sessions.

The main result of this experiment is that the targets display strategy preferred by participants (DS29) in user study 3, is also performing very well in terms of BCI performance, being similar to the control and optimal display strategy DS0. We hypothesize that, as the only common dimension between DS0 and DS29 is the size of the targets, the loss of performance in DS15 and DS22 is probably due the smaller-sized targets in these two conditions, which is also consistent with the literature [26].

In this experiment, two aspects were tested. First, the analysis of the questionnaires about the participants' preferences confirmed what was found in the previous experiment: condition DS29 was significantly the most preferred one. Secondly, the quantitative evaluation of the SSVEP classification accuracy shows that the most preferred display strategy (DS29) was not significantly worse than the control strategy (DS0).. Taken together our results confirm the recommendation to display the targets in the same plan as the robot's (horizontally) and with an exo-centerd frame-of-reference, as this is both preferred by the users and gives the same level of performance as more classical display strategies.

\section{Final Prototype}

The last step of our approach consisted in the development of a final prototype to illustrate and integrate our results in a unique setup. The application being the control of a mobile robot in AR by using a BCI and SSVEP-based commands. We consider here the use of a real mobile robot.

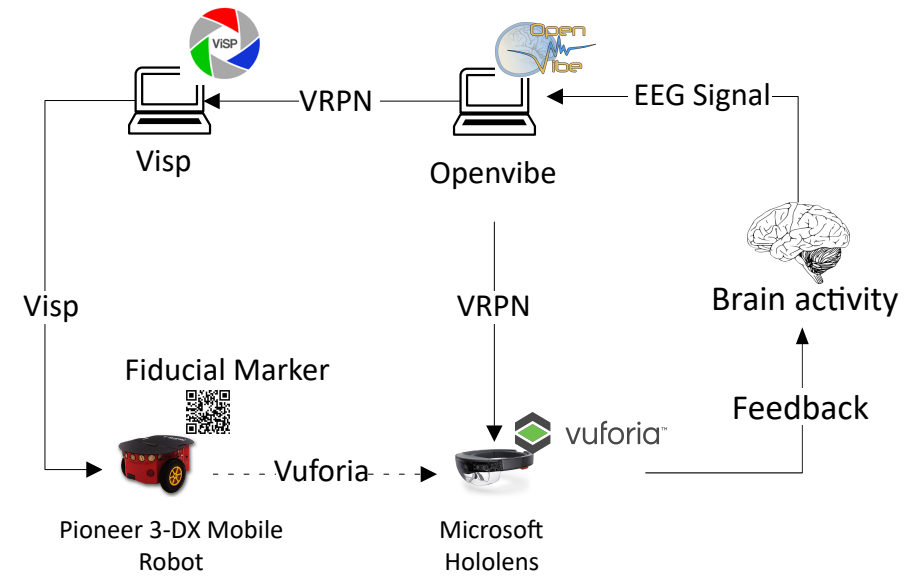

Fig. 11: Prototype architecture: Brain activity is measured through EEG and analyzed on a computer running the OpenvViBE software. The classification results are sent through VRPN to the HoloLens for feedback display and to a computer running Visp software for controlling the robot. The detected class is translated into a Visp command to make the robot move in the desired direction. A fiducial marker is placed on the Pioneer 3-DX robot to contextually make the flickering targets appear on the HoloLens when detected with Vuforia.

The general architecture of our final prototype is presented in Figure 11. Our system includes several hardware elements and software libraries. In addition to the g.tec EEG cap to record brain activity, we used the same Microsoft HoloLens headset. The robot used was a Pioneer 3-DX controlled by an additional computer through an application running a Visp-based [29] application. The hardware setup is presented in Figure 10. EEG data was analyzed on-line using OpenViBE which communicated with the Visp [29] application and the AR application running in the HoloLens through VRPN (Virtual-Reality Peripheral Network) [30] messages. The tracking of the fiducial marker on the robot was done using the Vuforia AR tracking library, compatible with the Universal Windows Platform of the Hololens.

The robot was controlled using the same three directional commands: (1) forward to make it go forward at a speed of $0.2 \mathrm{~m} / \mathrm{s}$, (2) rightward to make it do a rightward rotation of $15 \mathrm{deg} / \mathrm{s}$ and (3) leftward for the leftward rotation at $15 \mathrm{deg} / \mathrm{s}$. The commands consisted in three SSVEP targets. These targets were activated and displayed when the fiducial marker placed on top of the robot was in the field of view of the user. Focusing on one of the targets triggered its associated command. If the user was not focusing on any target the robot stopped (see Figure 10). The control of the prototype robot was continuous. Thus, the user was able to perform head movements while steering the robot. The layout of the targets could be done using any of the display strategies presented in our design space of the previous section. During our testing session, we used DSO.

We conducted informal testings with the real robots. A path was set up with 4 markers on the ground, and participants had to move the robot through each one of them. The targets were displayed following the DS0 display strategy. Informal testings showed that participants were well able to reasonably control the robot along the defined path, given the latency of SSVEP recognition $(\approx 7 s)$ (see the accompanying video). 


\section{Discussion}

Combining Brain-Computer Interfaces with Augmented Reality raises a lot of scientific and technological challenges. It poses theoretical questions regarding the design of effective interaction paradigms but it also involves feasibility studies regarding notably the compatibility of these two complex technologies. In addition, the design of AR-BCI systems is also strongly related to the chosen BCI paradigm and the designed UI. In the case of SSVEP it involves the placement of flickering targets in a comprehensive way. The design also relates to the final task/application and for instance to the number of possible commands. Designing an ARBCI application often requires: (1) testing hardware compatibility, depending on the EEG cap, the AR device and the exploited cortical area, (2) testing performance in real conditions (light, movement, etc), (3) designing the user interface related to all the BCI components (command semantics, stimulations and feedbacks) with respect to the final application and (4) validating the effect of the user interfaces on the BCI task performance.

Our paper has drawn and followed this line of research. In particular, our experiments aimed to study (1) whether it is possible to combine a binocular OST-HMD with a BCI without any significant drop of performance in SSVEP recognition, and (2) whether it is possible to tolerate head movements and thus select moving targets in AR. Our results showed that neither the wearing of the HMD, its electrical activity nor small head movements notably disrupted the BCI recognition accuracy. Thus, theoretically allowing us to use SSVEP in order to design user interfaces in AR. These studies also resulted in specific guidelines for our application as we made sure to set up the robot speed so that the movement it would require from the user would always remain below the maximum movement intensity corresponding to $40^{\circ}$ of amplitude.

In a second time, we proposed a design space involving 5 dimensions: (1) Orientation, (2) Frame-of-Reference, (3) Anchorage, (4) Size and (5) Explicitness. A qualitative evaluation (user study 3 ) of the 32 possible combinations (display strategies) provided us with guidelines on how users generally perceive the semantics of associating spatially displayed targets with commands. Most notably, the frame-of-reference set to the robot (exo-centered) helped them better understand which target corresponds to which direction. This could be explained by the spatial rotation task it requires to map targets and directions (represented by the wheels) when they are not in the same frame-of-reference. Similarly, a transversal orientation of the targets' plane was preferred to a frontal one. This could be explained by the 2D nature of the task, controlling a mobile robot. In other words, participants could have associated more easily the directions with the targets when the targets were in the same plane as the robot motion. On the other hand, a validation study (user study 4) of the SSVEP recognition performance using four representative and well ranked display strategies, confirmed the impact of the UI on the SSVEP recognition performance, notably regarding the size of the targets. These results demonstrate the importance of balancing the intuitiveness of the $\mathrm{UI}$ and the BCI command recognition performance. Thus, any application combining BCI and AR should take the trade-off between these two parameters into consideration.

The results of our different studies inspired the development of a prototype illustrating the combined use of $\mathrm{BCI}$ and $\mathrm{AR}$ to steer a mobile robot through a SSVEP interface in AR. Our prototype is functional and allowed us to test some of our 32 display strategies to control a virtual or real mobile robot.
Our approach provided a lot of insights on how to design BCIbased AR systems, it also raises numerous challenges to be tackled before such systems can be broadly used. First, future work should focus on developing efficient signal filtering methods and artifact removal procedures in order to allow wider and quicker movement while using BCIs and therefore to allow the selection of quicker moving targets. In addition, our goal in this paper was to be as exhaustive as possible on the characterization of the design space of target integration for 2D mobile object control. In future work, the question of integrating targets to control 3D moving objects (e.g. flying drones) or multi-function devices would be interesting and important to tackle, in order to make AR-BCI based interaction useful in other application cases.

Future studies could also explore other BCI paradigms and other fields of application for interaction in augmented reality environments. Between others, motor imagery [31] and covert attention [32], could benefit from the wideness of future OSTHMD's screens and constitute interesting paths. Motor-imagery based BCIs are typically used for controlling smart wheelchairs and enable severely impaired patients to regain in autonomy. It seems promising to couple such assistive technologies with AR systems in order to augment the environment and provide new hand-free interaction possibilities.

Nonetheless, in order to make BCI-based AR systems usable, it is not enough to improve the hardware and software components of the system. The user, and particularly their training, should also be considered. Thus, investigating the user experience, for instance through the development of new feedback modalities to keep the user engaged and to help them modulate their brain activity in AR, seems necessary, as well as to evaluate the extent to which users feel immersed and in control when interacting with a BCI/OST-HMD system. In this scope, it would be interesting to compare the combination of OST-HMDs and BCIs with other interaction technologies, both in terms of user experience and system performance.

\section{Conclusion}

In this paper we studied the combination of Brain-Computer Interfaces and Augmented Reality. We focused on one BCI category (BCIs based on SSVEP brain pattern), one AR category (optical see-through AR systems), and one task (mobile robot control).

First, we tested the feasibility of combining AR and BCI technologies and assessed the influence of external and internal noise on the BCI accuracy within two user studies. We first found that using an AR headset did not significantly impair the BCI performance and did not disrupt the EEG classification accuracy. Thus it seems possible to exploit EEG data with an AR headset upon the EEG cap. Second, we found that it is possible to use an SSVEP-based BCI in presence of small head movements.

Third, we proposed an extended design space for target integration in AR-SSVEP based applications. The display strategy relies on five dimensions: orientation, frame-of-reference, anchorage, size and explicitness. A third user study conducted with 42 participants enabled to identify and rank the most intuitive/coherent among 32 display strategies. This study notably showed that participants globally preferred when the frame-of-reference of the targets layout was set on the robot (rather than on the user) and when the targets plane was traversal (rather than frontal), i.e. parallel to the 2D robot motion. We also quantitatively studied the effect of the display strategies on the BCI performance. A fourth user study conducted 
with 24 participants showed that the targets' layout had an effect on the BCI performance. This suggests that the intuitiveness of the display strategy and the task performance have to be balanced.

Lastly, the paper illustrated the development of a complete and operational prototype for BCI-based control of a mobile robot in AR. Our final setup makes it possible to steer a real robot in a hands-free manner, opening perspectives for more realistic scenarios involving people with disabilities for instance.

Overall we have proposed and tested a scalable approach to guide the design of UIs based on BCIs in AR. We believe that such methodology could be adapted and reused in other contexts (e.g., other BCI categories, other AR/VR categories, other tasks) and could orient future research on BCI-based interactions in augmented and virtual reality.

\section{REFERENCES}

[1] J. R. Wolpaw and E. W. Wolpaw, "Brain-computer interfaces: something new under the sun," Brain-computer interfaces: principles and practice, pp. 3-12, 2012.

[2] H. Si-Mohammed, F. Argelaguet, G. Casiez, N. Roussel, and A. Lécuyer, "Brain-computer interfaces and augmented reality : A state of the art," in 7th International Brain-Computer Interface Conference, 2017.

[3] C. S. Herrmann, "Human eeg responses to $1-100 \mathrm{hz}$ flicker: resonance phenomena in visual cortex and their potential correlation to cognitive phenomena," Experimental brain research, vol. 137, no. 3-4, pp. 346-353, 2001.

[4] A. Evain, F. Argelaguet, N. Roussel, G. Casiez, and A. Lécuyer, "Can $i$ think of something else when using a bci?: Cognitive demand of an ssvep-based bci," in Proceedings of the 2017 CHI Conference on Human Factors in Computing Systems. ACM, 2017, pp. 5120-5125.

[5] J. Faller, B. Z. Allison, C. Brunner, R. Scherer, D. Schmalstieg, G. Pfurtscheller, and C. Neuper, "A feasibility study on ssvep-based interaction with motivating and immersive virtual and augmented reality," arXiv preprint arXiv:1701.03981, 2017.

[6] J. Faller, R. Leeb, G. Pfurtscheller, and R. Scherer, "Avatar navigation in virtual and augmented reality environments using an ssvep bci icabb-2010," in Proceedings of the Brain-Computer Interfacing and Virtual Reality Workshop W, vol. 1, 2010.

[7] P. Gergondet, S. Druon, A. Kheddar, C. Hintermüller, C. Guger, and M. Slater, "Using brain-computer interface to steer a humanoid robot," in 2011 IEEE International Conference on Robotics and Biomimetics (ROBIO). IEEE, 2011, pp. 192-197.

[8] E. Tidoni, P. Gergondet, G. Fusco, A. Kheddar, and S. M. Aglioti, "The role of audio-visual feedback in a thought-based control of a humanoid robot: A bci study in healthy and spinal cord injured people," IEEE Transactions on Neural Systems and Rehabilitation Engineering, vol. 25, no. 6, pp. 772-781, June 2017.

[9] C. Escolano, J. Antelis, and J. Minguez, "Human brain-teleoperated robot between remote places," in Robotics and Automation, 2009. ICRA'09. IEEE International Conference on. IEEE, 2009, pp. 4430-4437.

[10] A. Lenhardt and H. Ritter, "An augmented-reality based brain-computer interface for robot control," in International Conference on Neural Information Processing. Springer, 2010, pp. 58-65.

[11] L. A. Farwell and E. Donchin, "Talking off the top of your head: toward a mental prosthesis utilizing event-related brain potentials," Electroencephalography and clinical Neurophysiology, vol. 70, no. 6, pp. 510-523, 1988.

[12] E. Correa-Agudelo, A. M. Hernandez, C. Ferrin, and J. D. Gomez, "Vilimbs: Improving phantom limb treatment through multisensory feedback," in Proceedings of the 33rd Annual ACM Conference Extended Abstracts on Human Factors in Computing Systems, ser. CHI EA '15. ACM, 2015, pp. 1313-1318. [Online]. Available: http://doi.acm.org/10.1145/2702613.2732874
[13] V. S. Ramachandran, D. Rogers-Ramachandran, and S. Cobb, "Touching the phantom limb," Nature, vol. 377, no. 6549, p. 489, 1995.

[14] K. Takano, N. Hata, and K. Kansaku, "Towards intelligent environments: An augmented realitybrainmachine interface operated with a see-through head-mount display," Frontiers in Neuroscience, vol. 5, p. 60, 2011. [Online]. Available: http://journal.frontiersin.org/article/10.3389/fnins.2011.00060

[15] O. Friman, I. Volosyak, and A. Graser, "Multiple channel detection of steady-state visual evoked potentials for brain-computer interfaces," IEEE transactions on biomedical engineering, vol. 54, no. 4, pp. 742-750, 2007.

[16] X. Chen, Z. Chen, S. Gao, and X. Gao, "A high-itr ssvep-based bci speller," Brain-Computer Interfaces, vol. 1, no. 3-4, pp. 181-191, 2014.

[17] H.-J. Hwang, J.-H. Lim, Y.-J. Jung, H. Choi, S. W. Lee, and C.-H. Im, "Development of an ssvep-based bci spelling system adopting a qwertystyle led keyboard," Journal of neuroscience methods, vol. 208, no. 1, pp. 59-65, 2012.

[18] Y.-P. Lin, Y. Wang, C.-S. Wei, and T.-P. Jung, "Assessing the quality of steady-state visual-evoked potentials for moving humans using a mobile electroencephalogram headset," Frontiers in human neuroscience, vol. 8, 2014.

[19] Y. Wang, X. Gao, B. Hong, C. Jia, and S. Gao, "Brain-computer interfaces based on visual evoked potentials," IEEE Engineering in Medicine and Biology Magazine, vol. 27, no. 5, pp. 64-71, 2008.

[20] M. A. Pastor, J. Artieda, J. Arbizu, M. Valencia, and J. C. Masdeu, "Human cerebral activation during steady-state visual-evoked responses," Journal of neuroscience, vol. 23, no. 37, pp. 11 621-11 627, 2003.

[21] Y. Renard, F. Lotte, G. Gibert, M. Congedo, E. Maby, V. Delannoy, O. Bertrand, and A. Lécuyer, "Openvibe: An open-source software platform to design, test, and use brain-computer interfaces in real and virtual environments," Presence: teleoperators and virtual environments, vol. 19, no. 1, pp. 35-53, 2010.

[22] B. Blankertz, M. Kawanabe, R. Tomioka, F. U. Hohlefeld, V. V. Nikulin, and K.-R. Müller, "Invariant common spatial patterns: Alleviating nonstationarities in brain-computer interfacing." in NIPS, 2007, pp. 113120.

[23] K.-R. Müller, M. Tangermann, G. Dornhege, M. Krauledat, G. Curio, and B. Blankertz, "Machine learning for real-time single-trial eeg-analysis: from brain-computer interfacing to mental state monitoring," Journal of neuroscience methods, vol. 167, no. 1, pp. 82-90, 2008.

[24] M. Fatourechi, A. Bashashati, R. K. Ward, and G. E. Birch, "Emg and eog artifacts in brain computer interface systems: A survey," Clinical neurophysiology, vol. 118, no. 3, pp. 480-494, 2007.

[25] G. Müller-Putz, R. Scherer, C. Brunner, R. Leeb, and G. Pfurtscheller, "Better than random: a closer look on bci results," International Journal of Bioelectromagnetism, vol. 10, no. EPFL-ARTICLE-164768, pp. 52-55, 2008.

[26] K. B. Ng, A. P. Bradley, and R. Cunnington, "Stimulus specificity of a steady-state visual-evoked potential-based brain-computer interface," Journal of Neural engineering, vol. 9, no. 3, p. 036008, 2012.

[27] M. Balinski and R. Laraki, Majority judgment: measuring, ranking, and electing. MIT press, 2011.

[28] B. Allison, T. Luth, D. Valbuena, A. Teymourian, I. Volosyak, and A. Graser, "Bci demographics: How many (and what kinds of) people can use an ssvep bci?" IEEE transactions on neural systems and rehabilitation engineering, vol. 18, no. 2, pp. 107-116, 2010.

[29] É. Marchand, F. Spindler, and F. Chaumette, "Visp for visual servoing: a generic software platform with a wide class of robot control skills," IEEE Robotics \& Automation Magazine, vol. 12, no. 4, pp. 40-52, 2005.

[30] R. M. Taylor II, T. C. Hudson, A. Seeger, H. Weber, J. Juliano, and A. T. Helser, "Vrpn: a device-independent, network-transparent vr peripheral system," in Proceedings of the ACM symposium on Virtual reality software and technology. ACM, 2001, pp. 55-61.

[31] G. Pfurtscheller and C. Neuper, "Motor imagery and direct brain-computer communication," Proceedings of the IEEE, vol. 89, no. 7, pp. 1123-1134, 2001.

[32] L. Tonin, R. Leeb, A. Sobolewski, and J. del R Millán, "An online eeg bci based on covert visuospatial attention in absence of exogenous stimulation," Journal of neural engineering, vol. 10, no. 5, p. 056007 , 2013. 